\title{
Comparative Study of Xenobiotic-Free Media for the Cultivation of Human Limbal Epithelial Stem/Progenitor Cells
}

\author{
Sheyla González, PhD, Luxia Chen, MD, PhD, ${ }^{1,2}$ and Sophie X. Deng, MD, PhD ${ }^{1}$
}

The culture of human limbal epithelial stem/progenitor cells (LSCs) in the presence of animal components poses the risk of cross-species contamination in clinical applications. We quantitatively compared different xenobioticfree culture media for the cultivation of human LSCs. LSCs were cultured from $2 \times 2 \mathrm{~mm}$ limbal tissue explants on denuded human amniotic membrane with different xenobiotic-free culture media: CnT-Prime (CnT-PR) supplemented with $0 \%, 1 \%, 5 \%$, and 10\% human serum (HS), embryonic stem cell medium (ESCM) alone or in combination with the standard supplemented hormonal epithelium medium (SHEM, control) at a 1:1 dilution ratio, and modified SHEM (mSHEM), in which cholera toxin and dimethyl sulfoxide (DMSO) were removed, isoproterenol was added, and the epidermal growth factor concentration was reduced. Several parameters were quantified to assess the LSC phenotype: cell morphology, cell growth, cell size, outgrowth size, and expression of the undifferentiated LSC markers cytokeratin (K) 14, and p63 $\alpha$ high-expressing (p63 $\alpha^{\text {bright }}$ ) cells, a mature keratinocyte marker K12, epithelial marker pancytokeratin (PanK), and stromal cell marker vimentin (Vim). Compared with the standard SHEM control, CnT-PR base medium was associated with a lower cell growth and reduction in the proportion of stem cells generated regardless of the amount of HS supplemented $(p<0.05)$; ESCM resulted in an increased proportion of $\mathrm{PanK}^{-} / \mathrm{Vim}^{+}$stromal cells $(p<0.05)$ and a decreased proportion of p63 $\alpha^{\text {bright }}$ cells $(p<0.05)$; mSHEM supported a similar cell growth $(p>0.05)$, increased the number of small cells (diameter $\leq 12 \mu \mathrm{m} ; p<0.05$ ), and provided a similar proportion of $\mathrm{p} 63 \alpha^{\text {bright }}$ cells $(p>0.05)$. Among all the conditions tested, mSHEM was the most efficient and consistent in supporting the LSC phenotype and growth.

Keywords: limbal epithelial stem cells, limbal stem cell deficiency, limbal stem cell therapy, isoproterenol, CnT-Prime, embryonic stem cell medium, SHEM, xenobiotic-free

\section{Introduction}

$\mathbf{L}$ IMBAL STEM CELL DEFICIENCY (LSCD) is a corneal disorder, in which limbal epithelial stem/progenitor cells (LSCs) are absent or dysfunctional and, consequently, the ability to regenerate a corneal epithelial surface is lost. LSCD is characterized by persistent epithelial defects, conjunctivalization, neovascularization, scarring, and inflammation, all of which lead to corneal opacity, pain, photophobia, and ultimately blindness.

Corneal transplantation is ineffective to treat severe to total LSCD because functional LSCs are not transplanted. The highest success rate treating LSCD was achieved by using cultivated LSCs that were expanded from single LSCs cultured on $3 \mathrm{~T} 3$ mouse fibroblasts feeder layers in culture medium that contained fetal bovine serum (FBS). ${ }^{1}$ However, the presence of animal components in the culture system posed the risk of transmitting animal diseases to human recipients of these LSCs.

Different formulations of xenobiotic-free culture media that can sustain the growth of LSCs in vitro have been used, but their clinical success rates were reported to be lower than that using 3T3 feeder cells and bovine fetal serum. ${ }^{2-5}$ For instance, transplantation of cultivated LSCs under xenobiotic-free explant culture supplemented with autologous serum achieved $71 \%$ clinical success. ${ }^{2}$ Anatomical

\footnotetext{
${ }^{1}$ Stein Eye Institute, University of California, Los Angeles, California.

${ }^{2}$ Tianjin Eye Hospital and Eye Institute, Tianjin, China.

(C) Sheyla González, et al., 2017; Published by Mary Ann Liebert, Inc. This Open Access article is distributed under the terms of the Creative Commons Attribution Noncommercial License (http://creativecommons.org/licenses/by-nc/4.0/) which permits any noncommercial use, distribution, and reproduction in any medium, provided the original author(s) and the source are credited.
} 
Table 1. Media Conditions Tested

\begin{tabular}{lll}
\hline \multicolumn{3}{c}{ Media conditions } \\
\hline \multicolumn{1}{c}{ CnT-PR (Explant), $\mathrm{n}=4$} & ESCM (Explant), $\mathrm{n}=6$ & \multicolumn{1}{c}{ SHEM (Explant), $\mathrm{n}=3$} \\
\hline CnT-PR 0 (CnT-PR w/o HS) & ESCM 1 (ESCM) & SHEM 1 (SHEM-Chol-DMSO) \\
CnT-PR 1 (CnT-PR 1\%HS) & ESCM 2 (SHEM:ESCM) & SHEM 2 (SHEM10-Chol-DMSO) \\
CnT-PR 5 (CnT-PR 5\%HS) & & SHEM 3 (SHEM-Chol-DMSO+Ipr) \\
CnT-PR 10 (CnT-PR 10\%HS) & & SHEM 4 (SHEM-Chol+DMSO+Ipr) \\
& & SHEM 5 (SHEM-Chol-DMSO+Ipr+1XEGF) \\
\hline
\end{tabular}

3T3 SHEM Cntl

Explant SHEM Cntl

Italics are the most efficient culture media for the growth of the LSCs.

Chol, cholera toxin; Cntl, control; CnT-PR, CnT-Prime, epithelial culture medium; DMSO, dimethyl sulfoxide; EGF, epithermal growth factor; ESCM, embryonic stem cell medium; Expl, explant; HS, human serum; Ipr, isoproterenol/Isuprel; LSCs, limbal epithelial stem/ progenitor cells; SHEM, supplemented hormonal epithelium medium.

restoration of a corneal epithelial surface was achieved in $67 \%$ of patients who received cultivated LSCs using CnT-Prime (CnT-PR) medium (formerly CnT-20) supplemented with human serum (HS) in a Phase I/II trial. ${ }^{4,5}$ Embryonic stem cell medium (ESCM) supplemented with KnockOut Serum Replacement has also been used to culture LSCs in vitro. ${ }^{6}$ FBS from supplemented hormonal epithelium medium (SHEM) has been replaced by KnockOut Serum Replacement in the LSC culture. ${ }^{7}$ Also, SHEM has been further modified to replace cholera toxin with isoproterenol in vitro. ${ }^{8}$ However, the efficiency of these different xenobiotic-free media in supporting the expansion of LSCs has not been compared directly using the same criteria.

It has been shown that the percentage of p63 $\alpha$ highexpressing $\left(\mathrm{p} 63 \alpha^{\text {bright }}\right)$ cells correlated with clinical success. ${ }^{1}$ In the present study, we investigated the efficiency of different xenobiotic-free culture media, with measures of efficiency defined as cell growth and the relative amount of stem/progenitor cells, including $\mathrm{p} 63 \alpha^{\text {bright }}$ cells, generated in each culture medium.

\section{Materials and Methods}

\section{Limbal epithelial stem cell culture}

The study was exempted by the University of California Los Angeles Institutional Review Board (IRB No. 11-003284 and IRB No. 12-000363). Human sclerocorneal tissues from 40- to 70-year-old donors were obtained from eye banks and handled in accordance with the tenets of the Declaration of Helsinki. The tissues were preserved in Optisol ${ }^{\mathrm{TM}}$ (Chiron Ophthalmics, Inc., Irvine, CA) or Life4C (Numedis, Inc., Isanti, MN) at $4^{\circ} \mathrm{C}$. The death-to-preservation time was less than $12 \mathrm{~h}$, and the death-to-experiment time was less than 7 days. Amniotic membrane (AM) was selected as the culture substrate. Epithelial cells from the AM were mechanically removed as previously described ${ }^{9}$ in an EDTA solution (Versene; Life Technologies, Carlsbad, CA).

To isolate limbal epithelial cell sheets, the iris, endothelium, conjunctiva, and Tenon's capsule were removed from the sclerocorneal rim tissue. The rim was incubated in $2.4 \mathrm{U} /$ $\mathrm{mL}$ of dispase II (Roche, Indianapolis, IN) at $37^{\circ} \mathrm{C}$ for $2 \mathrm{~h}$ in Dulbecco's Modified Eagle's Medium (DMEM)/F-12 (Ham) medium (Life Technologies) followed by a gentle scrapping under the dissecting microscope. Single cells were obtained by incubation in $0.25 \%$ trypsin-EDTA (Life Technologies) for $5 \mathrm{~min}$. Explant tissue pieces (dimensions, $2 \times 2 \mathrm{~mm}$ ) were also obtained from the limbal area. Experiments were performed with three to six sclerolimbal donor tissues. LSCs were cultured by using limbal explants placed on denuded AM in different culture media (Table 1).

CnT-PR (CELLnTEC, Switzerland) was tested in the absence (CnT-PR 0) or presence of human serum (HS) (Innovative Research, Novi, MI); the concentrations of HS that were tested were 1\% (CnT-PR 1), 5\% (CnT-PR 5), and 10\% (CnT-PR 10; Table 1). ESCM 1 was prepared as previously described $^{6}$ with KnockOut DMEM (Life Technologies) supplemented with $10 \%$ KnockOut Serum Replacement (Life Technologies), $1 \%$ (v/v) N2 supplement (Life Technologies), $4 \mathrm{ng} / \mathrm{mL}$ of basic fibroblast growth factor (Life Technologies), $1 \mathrm{mM}$ L-glutamine, $0.1 \mathrm{mM} \beta$-mercaptoethanol (Life Technologies), and 1\% (v/v) nonessential amino acids (Life Technologies). ESCM 2, which consisted of ESCM and SHEM at a 1:1 ratio (Table 1), was also tested.

The standard SHEM was prepared as previously described $^{10}$ and used as a control medium. In brief, SHEM consisted of DMEM/F-12 medium supplemented with 5\% $\mathrm{HS}, 1 \%(\mathrm{v} / \mathrm{v}) \mathrm{N} 2$ supplement, $2.0 \mathrm{ng} / \mathrm{mL}$ of epidermal growth factor (EGF; Life Technologies), $8.4 \mathrm{ng} / \mathrm{mL}$ of cholera toxin (Sigma-Aldrich, St. Louis, MO), $0.5 \mu \mathrm{g} / \mathrm{mL}$ of hydrocortisone (Sigma-Aldrich), and $0.5 \%$ (v/v) of dimethyl sulfoxide (DMSO; Sigma-Aldrich).

Several modifications were made to SHEM (Tables 1 and 2): SHEM 1 was supplemented with 5\% HS, and both cholera toxin and DMSO were not added to the medium. In SHEM 2, HS was increased to $10 \%$. In SHEM 3, cholera toxin was replaced by isoproterenol ${ }^{8}$ (Sigma-Aldrich); the final concentration of isoproterenol was $1 \mu \mathrm{g} / \mathrm{mL}$. In SHEM

Table 2. SHEM Media Composition

\begin{tabular}{lrcccc}
\hline & & $E G F$ & & & \\
& $H S(\%)$ & $(n g / m L)$ & DMSO & Chol & Ipr \\
\hline Explant SHEM Cntl & 5 & 2.0 & Yes & Yes & No \\
SHEM 1 & 5 & 2.0 & No & No & No \\
SHEM 2 & 10 & 2.0 & No & No & No \\
SHEM 3 & 5 & 2.0 & No & No & Yes \\
SHEM 4 & 5 & 2.0 & Yes & No & Yes \\
SHEM 5 & 5 & 0.4 & No & No & Yes \\
\hline
\end{tabular}


4, DMSO and isoproterenol were added to the medium, but cholera toxin was not. Finally, in SHEM 5, the concentration of EGF was reduced to $0.4 \mathrm{ng} / \mathrm{mL}$; the rest of the components and their concentrations were the same as those in SHEM 3.

Single LSCs on growth-arrested 3T3 mouse fibroblasts (the Howard Green laboratory, Harvard Medical School) cultured in the standard unmodified SHEM with 5\% FBS (Life Technologies) served as the control (3T3 SHEM Cntl) for all tissue donors tested. Explants on denuded AM (Explant SHEM Cntl) cultured in unmodified SHEM with 5\% HS also served as another control of the LSC growth (Table 1). LSCs were cultured at $37^{\circ} \mathrm{C}$ with $5 \% \mathrm{CO}_{2}$ for up to 14 days. The medium was changed every 2-3 days.

\section{Analysis of cell growth, cell size, cell morphology, and transplantability of outgrowths}

Cell growth from a single explant tissue piece was calculated by counting the total number of cells harvested once the cells have been detached from the AM after dispase/ trypsin-EDTA treatment.

Cell size was measured by ImageJ software (Colony Counter plugin). The percentage of cells whose diameter was $\leq 12 \mu \mathrm{m}$ (small cells) was calculated for each culture condition.

Cell morphology of the outgrowths was assessed by using an inverted DMIL LED microscope (Leica Microsystems, Wetzlar, Germany). Cell images were taken with an Insight 11.2 color mosaic digital camera (Spot Imaging Solutions, Sterling Heights, Michigan). The outgrowth shape and size were measured; an outgrowth that was at least $13 \mathrm{~mm}$ in its smallest diameter after 10-14 days in culture was considered to be transplantable.

\section{Immunocytochemistry}

Cultured LSCs were treated with trypsin to generate a single-cell suspension that was subjected to cytocentrifugation (Cytofuge; Thermo Scientific, Waltham, MA) onto glass slides (Fisher Scientific, Canoga Park, CA), and stored at $-20^{\circ} \mathrm{C}$. Cells were fixed in a $4 \%$ paraformaldehyde solution (Electron Microscopy Sciences, Hatfield, PA). Blocking and permeabilization were performed in a solution of $1 \%$ bovine serum albumin (Sigma-Aldrich) and $0.5 \%$ Triton X-100 (Sigma-Aldrich) for $30 \mathrm{~min}$. Primary antibody incubation was done in a solution of $1 \%$ bovine serum albumin and $0.1 \%$ Triton $\mathrm{X}-100$, overnight at $4^{\circ} \mathrm{C}$ (antibodies are listed in Table 3). Secondary antibody incubation was done at room temperature for $1 \mathrm{~h}$. Nuclei were stained with Hoechst 33342 (Life Technologies) at room temperature for $10 \mathrm{~min}$. Mounting was done with Fluoromount medium (Sigma-Aldrich). Pictures were taken with a Zeiss Imager.A2 fluorescent microscope (Carl Zeiss, Inc., Oberkochen, Germany) equipped with an Insight 11.2 color mosaic digital camera.

Cells that expressed high levels of p63 $\alpha$ (p63 $\alpha^{\text {bright }}$ cells) were quantified by using the Definiens Tissue Studio software (Larchmont, NY) as previously reported. ${ }^{11}$ Quantitation of cells that expressed cytokeratin (K) 14, K12, pancytokeratin (PanK), or vimentin (Vim) was performed using ImageJ software.
Table 3. Primary Antibodies Used FOR IMMUNOCYTOCHEMISTRY

\begin{tabular}{lrl}
\hline $\begin{array}{l}\text { Primary } \\
\text { antibody }\end{array}$ & Dilution & \multicolumn{1}{c}{ Source (catalogue no.) } \\
\hline K12 & $1: 100$ & Santa Cruz Biotechnology (sc-25722) \\
K14 & $1: 50$ & Fisher Scientific (MS-115-R7) \\
p63 $\alpha$ & $1: 100$ & Cell Signaling Technology (No. 4892) \\
PanK & $1: 100$ & DAKO (M3515) \\
Vim & $1: 100$ & Abcam (ab92547) \\
\hline
\end{tabular}

K12, cytokeratin 12; K14, cytokeratin 14; PanK, pancytokeratin; Vim, vimentin.

\section{Statistical analysis}

Data were analyzed with the pairwise $t$-test. In the graphs, bars indicate the mean \pm standard error of the mean (SEM). $p$ Values $<0.05$ indicate statistical significance.

\section{Results}

\section{Efficiency of three different base media in supporting LSC growth}

We first investigated the optimal formulation for each of the three base media: CnT-PR, ESCM, and SHEM (Figs. 1-3 and Tables 1-2). After the optimal formulation of each base medium was determined, all three base media were compared to determine which one sustained the most efficient LSC growth (Figs. 4 and 5 and Table 4).

Outgrowths of undifferentiated LSCs contained small and cuboidal epithelial-like cells in the control cultures: 3T3 SHEM Cntl and Explant SHEM Cntl (Fig. 1A). LSC cultures in CnT-PR medium supplemented with different concentrations of HS showed variable and inconsistent results (Fig. 1). LSCs cultured in CnT-PR exhibited heterogeneous morphology; in some cases, the cells were spindle shaped. A large quantity of floating dead cells and loose cell sheets at the edge was present. The increased concentration of HS in CnT-PR 5 appeared to reduce the quantity of floating dead cells and led to a more compact LSC morphology (Fig. 1A). The cell expansion rate was significantly reduced in all CnT-PR conditions $(p<0.05)$, but the cell expansion rate in the CnT-PR culture with $10 \%$ HS $(p=0.05)$ was comparable to that of the explant SHEM Cntl in unmodified SHEM (Fig. 1B).

Analysis of the cell phenotype and quantitation of small cells (Fig. 1C) revealed that CnT-PR 5 was the most efficient of the CnT-PR-based media to culture LSCs. Compared with the explant SHEM Cntl, the culture grown in CnT-PR 5 produced a similar proportion of small cells $(\leq 12 \mu \mathrm{m}$; $3.5 \% \pm 1.0 \%$ in the control vs $3.9 \% \pm 1.7 \%, p=0.84$ ), a small proportion of differentiated $\mathrm{K}_{1} 2^{+}$cells $(1.5 \% \pm 0.4 \%$ in the control vs $2.7 \% \pm 1.1 \%, p=0.19)$, a high proportion of $\mathrm{PanK}^{+}$ epithelial cells $(98.8 \% \pm 0.4 \%$ in the control vs $96.9 \% \pm 0.9 \%$, $p=0.78$ ), and a similar proportion of $\mathrm{Vim}^{+}$stromal cells $(1.1 \% \pm 0.2 \%$ in the control vs $1 \% \pm 0.5 \%, p=0.50)$. The percentage of $\mathrm{p} 63 \alpha^{\text {bright }}$ cells, which has been previously positively correlated with the transplantation success, ${ }^{1}$ was the highest in CnT-PR 5 among all CnT-PR conditions tested $(p<0.05)$ and statistically comparable to the explant SHEM Cntl $(17.8 \% \pm 5.7 \%$ vs $27.6 \% \pm 5.2 \%$, respectively, $p=0.25)$. 
A

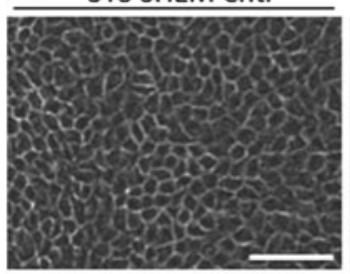

Explant SHEM Cntl

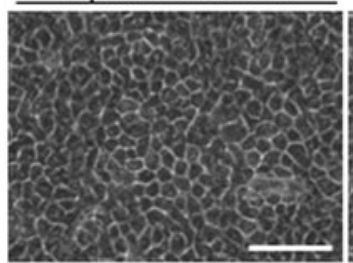

B

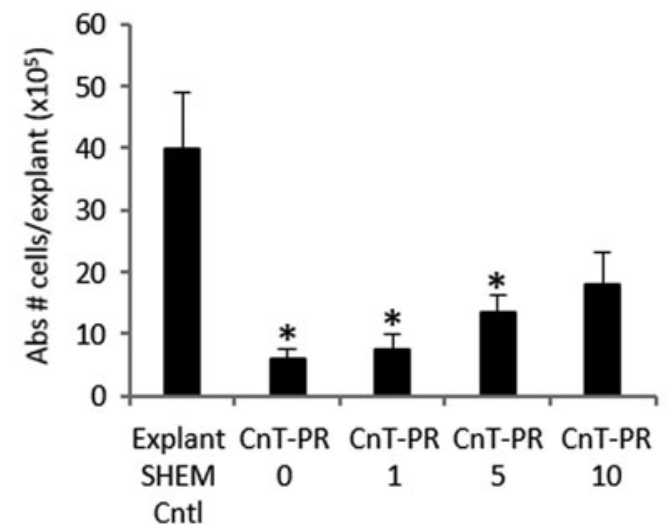

CnT-PR 0

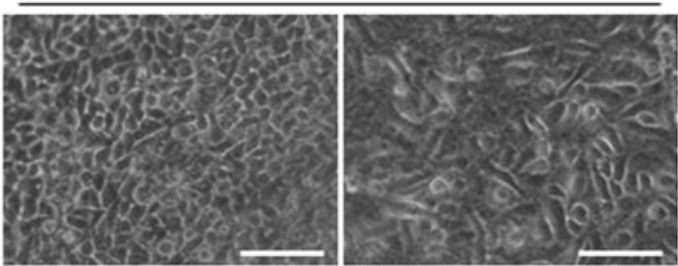

CnT-PR 5
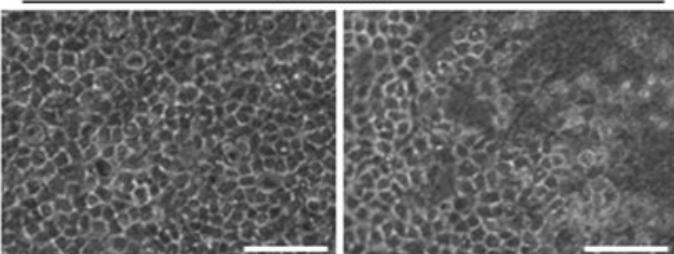

CnT-PR 1

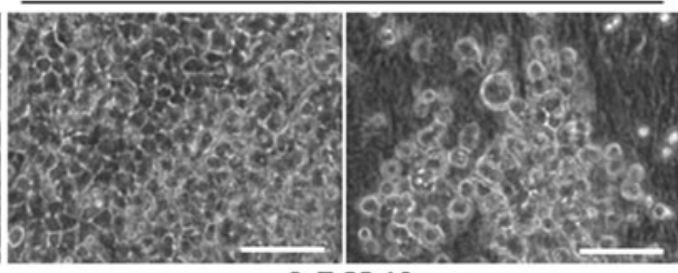

CnT-PR 10

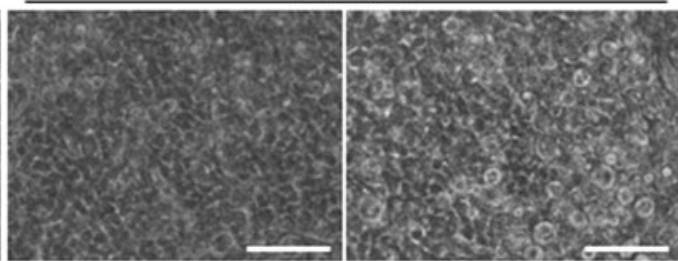

C Explant SHEM Cntl|r CnT-PR 0 CnT-PR 1 日CnT-PR 5 QCnT-PR 10

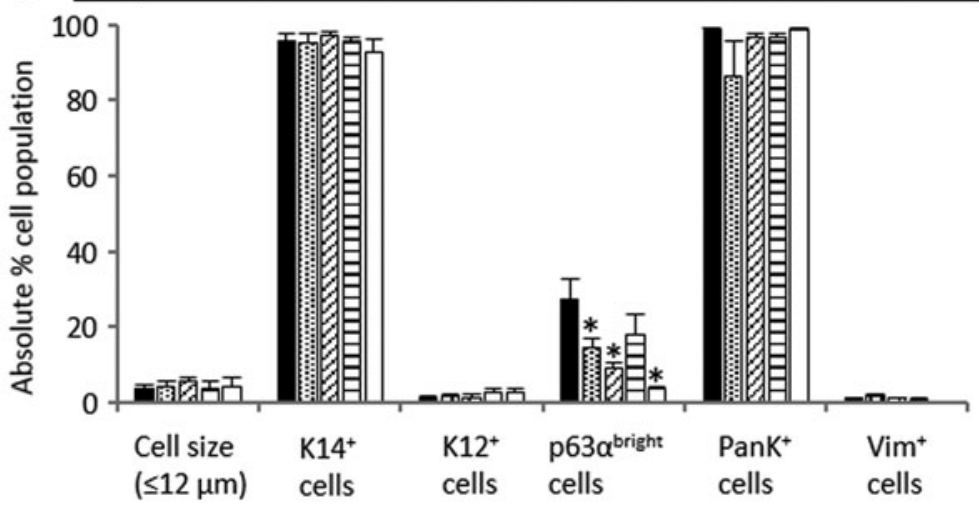

FIG. 1. Morphology (A), cell growth (B), and characterization (C) of LSCs cultured in four different CnT-PR conditions. "Abs \# cells/explant" refers to the absolute number of cells harvested from one explant tissue piece on average. Scale bar $50 \mu \mathrm{m}$. Two pictures of each culture medium condition have been included to show different areas of the outgrowth. ${ }^{*} p<0.05$ were considered significant. CnT-PR, CnT-Prime; LSCs, limbal epithelial stem/progenitor cells.
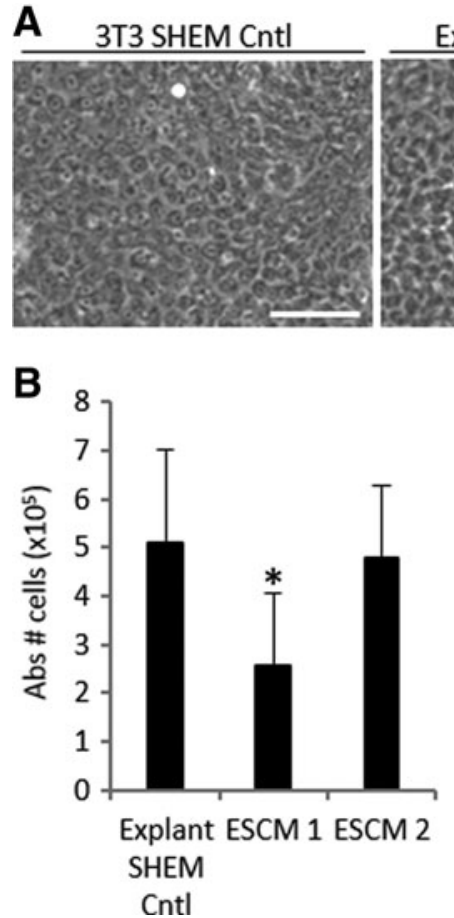

Explant SHEM Cntl

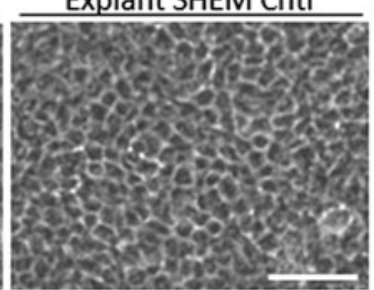

ESCM 2
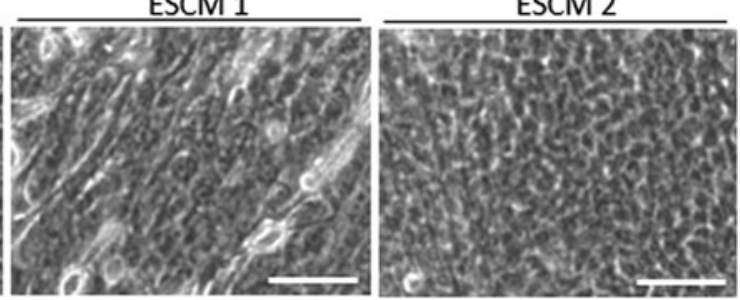

C

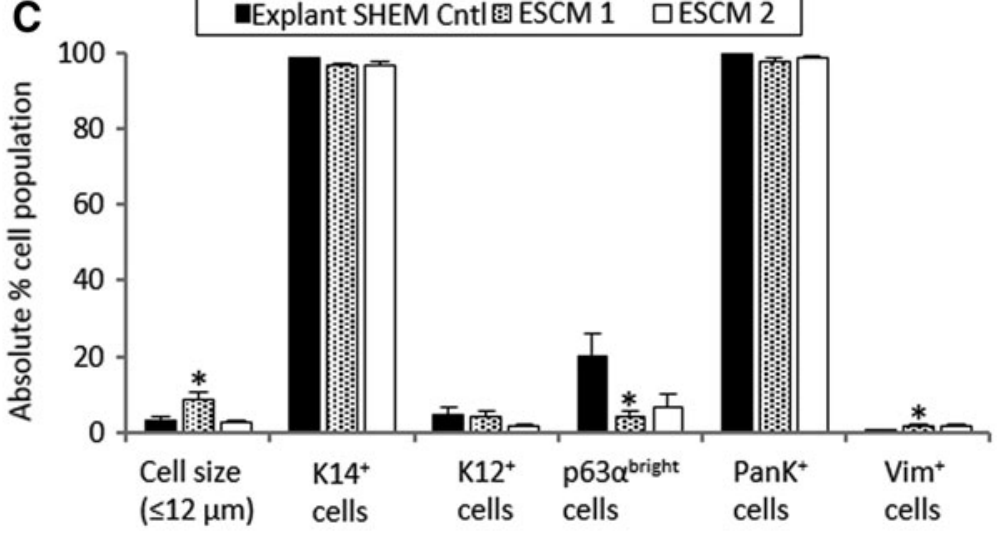

FIG. 2. Morphology (A), cell growth (B), and characterization (C) of LSCs cultured in two different ESCM conditions. "Abs \# cells/explant" refers to the absolute number of cells harvested from one explant tissue piece on average. Scale bar $50 \mu \mathrm{m} . * p<0.05$ were considered statistically significant. ESCM, embryonic stem cell medium. 


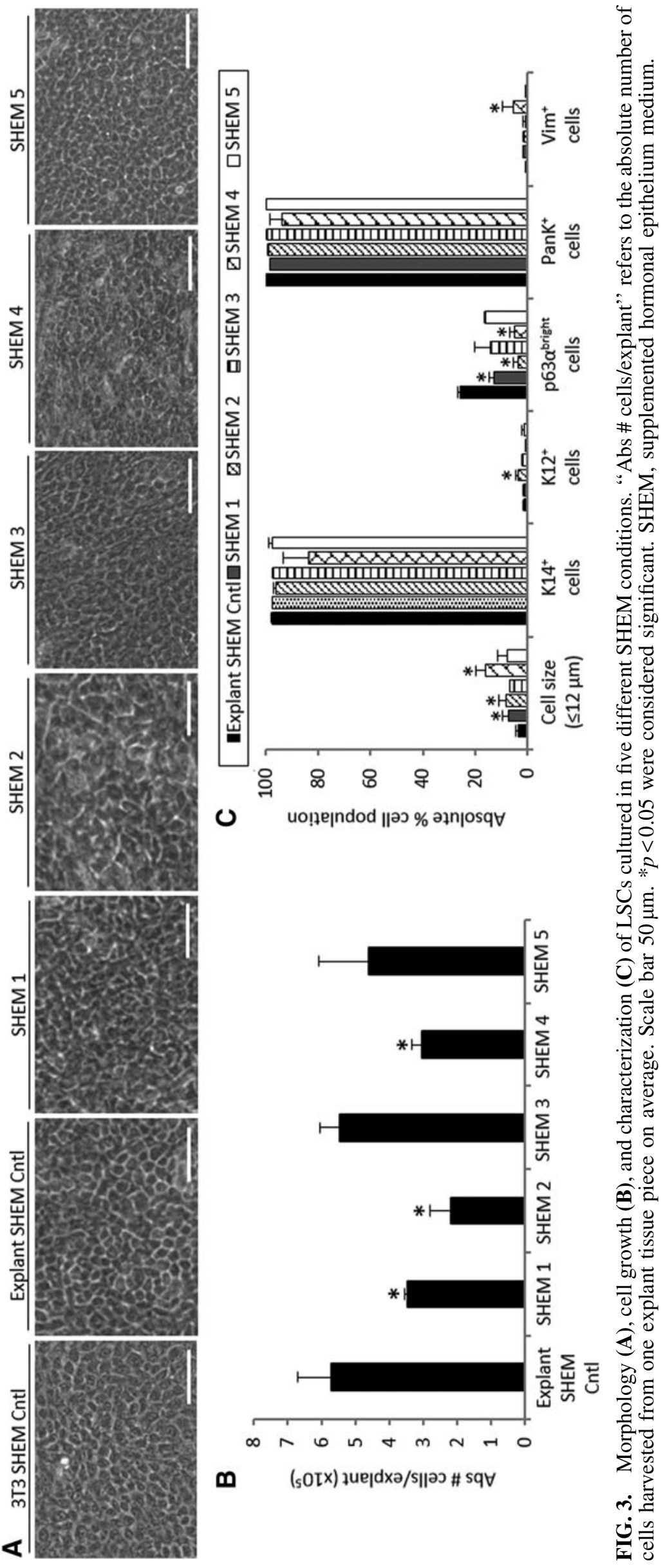



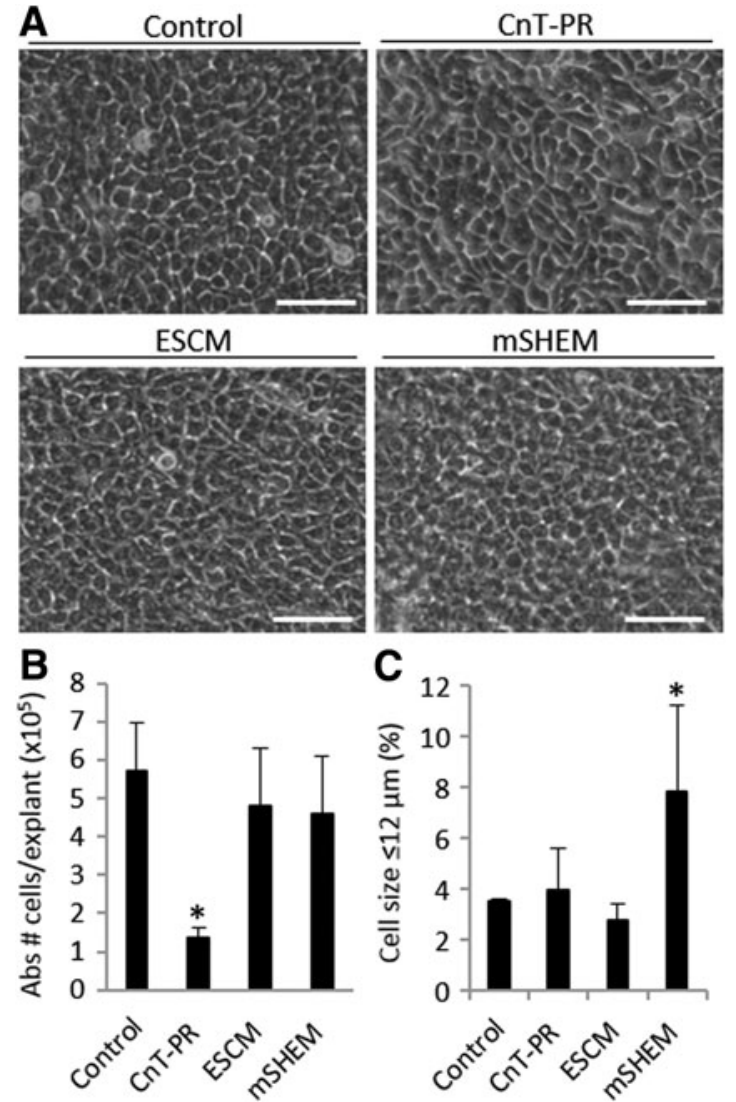

FIG. 4. Cell morphology (A), cell growth (B), and percentage of small cells $(\leq 12 \mu \mathrm{m})(\mathbf{C})$ among LSCs cultured in each base medium: CnT-PR, ESCM, and mSHEM. "Abs \# cells/explant" refers to the absolute number of cells harvested from one explant tissue piece on average. Scale bar $50 \mu \mathrm{m} .{ }^{*} p<0.05$ were considered significant.

LSCs cultured in ESCM 1 had a heterogeneous morphology consisting of a mixture of small and cuboidal epithelial-like and fibroblast-like cells (Fig. 2A). LSC cultures in ESCM 2 shared a similar morphology as did those in the 3T3 SHEM Cntl and Explant SHEM Cntl (Fig. 2A). The cell expansion rate in ESCM 1 was significantly lower than that in the explant SHEM Cntl (49.7\% slower in ESMC 1, $p=0.01$; Fig. 2B). In contrast to ESCM 1, ESCM 2 increased the LSC growth to a similar degree as in the explant SHEM Cntl ( $p=0.81$; Fig. 2B).

ESCM 1 increased the proportion of $\mathrm{Vim}^{+}$stromal cells $(1.8 \% \pm 0.6 \%$ vs $0.2 \% \pm 0.1 \%$ in the explant SHEM Cntl, $p=0.04)$ and the percentage of small cells $(8.4 \% \pm 2.2 \% \mathrm{vs}$ $3.3 \% \pm 0.9 \%$ in the explant SHEM Cntl, $p=0.04)$ and decreased the percentage of p63 $\alpha^{\text {bright }}$ cells $(4.4 \% \pm 1.3 \%$ vs $20.3 \% \pm 5.7 \%, p=0.04$; Fig. 2C). Compared with the explant SHEM Cntl, ESCM 2 provided a statistically similar percentage of $\mathrm{Vim}^{+}$cells $(1.6 \% \pm 0.8 \%, p=0.16), \mathrm{K}^{+} 2^{+}$cells $(1.8 \% \pm 0.6 \%, p=0.06)$, and p63 $\alpha^{\text {bright }}$ cells $(6.7 \% \pm 3.6 \%$, $p=0.06$; Fig. 2C).

When SHEM was modified, the substitution of cholera toxin by isoproterenol in the absence of DMSO (SHEM 3 and SHEM 5) improved the cell sheet quality by reducing the amount of floating dead cells and the heterogeneous morphology seen in cultures grown in other SHEM formu- lations. Cultures in the presence of SHEM 5, with a reduced concentration of EGF, maintained the limbal epithelial morphology (Fig. 3A). The cell expansion rate was significantly reduced in most of the modified SHEM (mSHEM) formulations $(p<0.05)$ except SHEM 3 and SHEM 5, in which cholera toxin and DMSO were replaced by isoproterenol (both $p \geq 0.05$; Fig. 3B).

LSCs cultured in SHEM 3 and SHEM 5 showed a phenotype similar to that of cells grown in the explant SHEM Cntl in terms of the percentage of small cells, p63 $\alpha^{\text {bright }}$ cells, $\mathrm{K}_{12}{ }^{+}$cells, and $\mathrm{Vim}^{+}$stromal cells (all $p>0.05$; Fig. 3C). However, other modifications of SHEM resulted in either a significant increase in the proportion of $\mathrm{K}_{1} 2^{+}$cells or $\mathrm{Vim}^{+}$cells, or a decrease in the percentage of p63 $\alpha^{\text {bright }}$ cells. Therefore, SHEM 5 appeared to be the best formulation among all 5 SHEM-based media.

\section{Growth of LSCs in the different optimized base media}

As shown above, CnT-PR 5, ESCM 2, and SHEM 5 were the most efficient culture media for the growth of LSCs (Table 1, italics). These conditions were compared in a second set of experiments and will be referred to as CnTPR, ESCM, and mSHEM, respectively, for simplicity in the rest of the article (Figures 4 and 5 and Table 4). Explant culture using the standard SHEM served as the control in these experiments and therefore will be referred to as the control.

We found that mSHEM supported the most compact and homogeneous cell outgrowths that contained small and cuboidal limbal epithelial-like cells (Fig. 4A). LSC cultures in CnT-PR and ESCM tended to be slightly more heterogeneous and variable (Fig. 4A).

ESCM and mSHEM supported the LSC growth similar to that of the explant SHEM Cntl ( $p>0.05$; Fig. 4B). However, the LSC growth in CnT-PR was $76.6 \%$ reduced compared with the explant SHEM Cntl ( $p=0.03$; Fig. 4B).

A higher percentage of small cells $(\leq 12 \mu \mathrm{m})$ was found in the mSHEM cultures than in other conditions, including explant SHEM Cntl $(7.8 \% \pm 3.4 \%$ vs $3.5 \% \pm 0.1 \%$, respectively, $p=0.04$; Fig. 4C). Both CnT-PR and ESCM were able to produce a similar percentage of small cells as the explant SHEM Cntl $(3.9 \% \pm 1.7 \%$ and $2.8 \% \pm 0.6 \%$, respectively; both $p>0.05$; Fig. 4C).

LSC outgrowths generated in each culture condition were classified as transplantable/nontransplantable on the basis of the size and shape of the cell sheet. Outgrowths that measured at least $13 \mathrm{~mm}$ in their smallest diameter were considered transplantable (Table 4). Cells grown in CnT-PR produced the smallest outgrowths and the smallest relative quantity of transplantable cell sheets $(33.3 \%$ vs $94.1 \%$ in the control, $p=0.02$; Table 4). In the ESCM cultures, the outgrowths were larger, and $62.5 \%$ were considered transplantable ( $p=0.03$; Table 4$)$. In comparison with ESCM and CnT-PR, mSHEM produced the most homogeneous outgrowths, and $100 \%$ were considered to be transplantable. On average, both diameters of cell outgrowths in mSHEM exceeded the 13-mm requirement (Table 4).

We next characterized the cell population in the cultures. The expression of K14 and K12 protein was analyzed to distinguish the proportion of undifferentiated corneal epithelial cells from the differentiated ones. Outgrowths from 


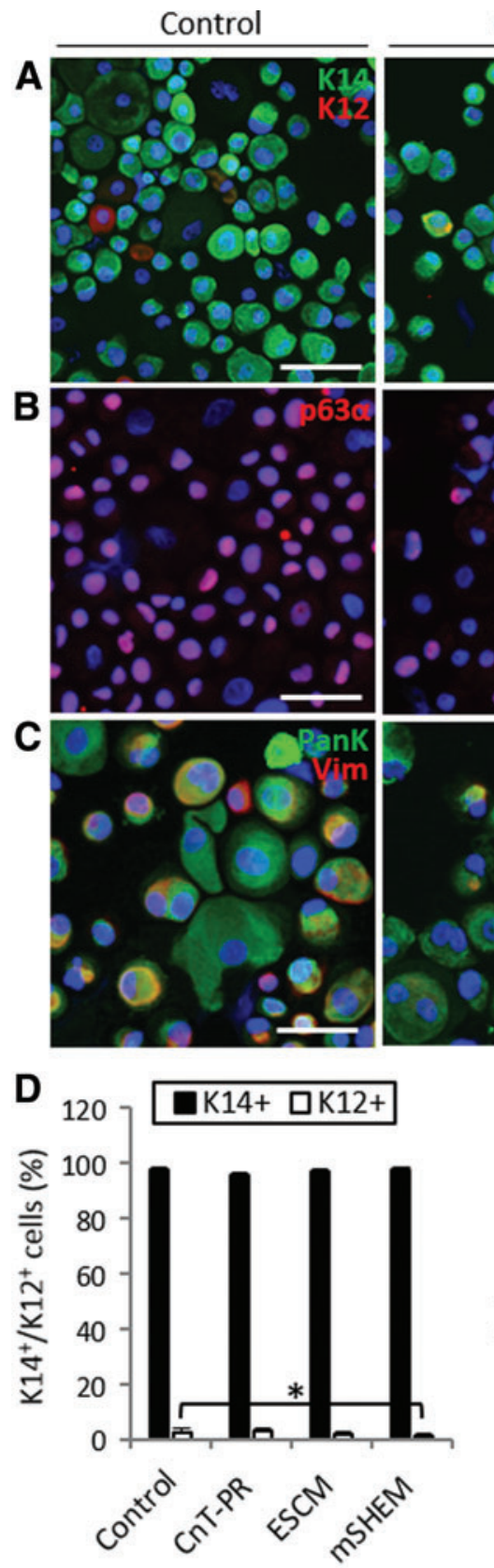

CnT-PR
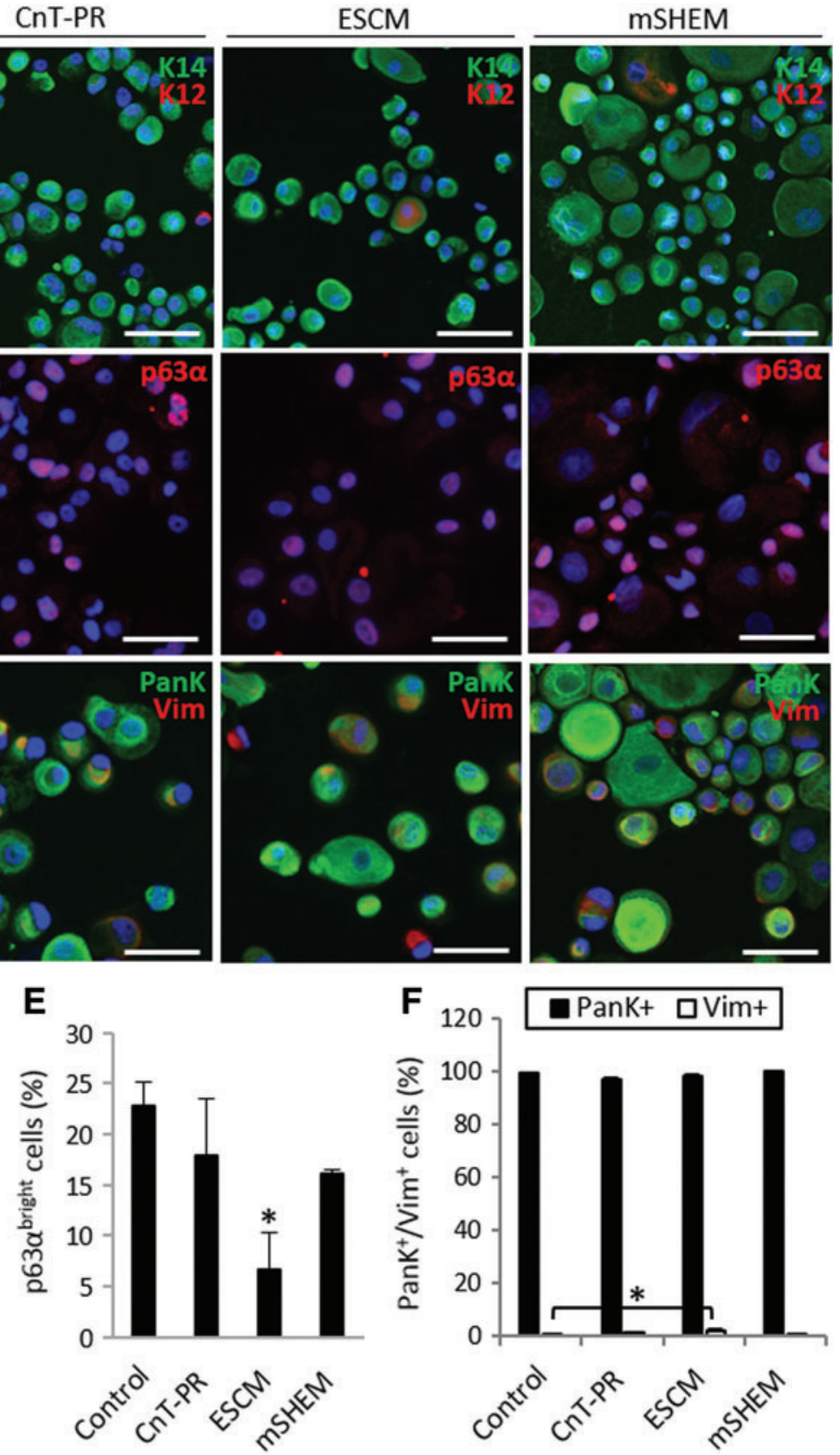

FIG. 5. Characterization of LSCs cultured in CnT-PR, ESCM, and mSHEM. Representative images of double immunostaining for the detection of K14-K12 (A), p63 $\alpha$ (B), and PanK-Vim (C). Scale bar $50 \mu$ m. Percentage of K14 $/ \mathrm{K} 12^{+}$cells (D), p63 $\alpha^{\text {bright }}$ cells (E), and $\mathrm{PanK}^{+} / \mathrm{Vim}^{+}$cells $(\mathbf{F}) .{ }^{*} p<0.05$ were considered significant. Color images available online at www.liebertpub.com/tec

Table 4. Size and Transplantability OF THE OUTGROWTHS IN THE DIFFERENT Media Conditions

\begin{tabular}{lccc}
\hline & $\begin{array}{c}\text { Major } \\
\text { diameter } \\
(\mathrm{mm})\end{array}$ & $\begin{array}{c}\text { Minor } \\
\text { diameter } \\
(\mathrm{mm})\end{array}$ & $\begin{array}{c}\text { Transplantable } \\
\text { outgrowths }(\%)\end{array}$ \\
\hline Control & $26.9 \pm 3.9$ & $21.4 \pm 1.7$ & 94.1 \\
CnT-PR & $13.5 \pm 3.2$ & $11.0 \pm 2.5$ & 33.3 \\
ESCM & $27.3 \pm 2.9$ & $13.2 \pm 2.0$ & 62.5 \\
mSHEM & $25.0 \pm 0.0$ & $23.0 \pm 2.0$ & 100.0 \\
\hline
\end{tabular}

mSHEM, modified SHEM. all three conditions contained a similar percentage of $\mathrm{K}^{+} 4^{+}$ cells, which was $>95 \%$ ( $p>0.05$; Fig. 5A, D). Only cultures grown in mSHEM contained a significantly smaller proportion of differentiated $\mathrm{K}_{1} 2^{+}$cells $(1.3 \% \pm 0.5 \%$ vs $2.5 \% \pm 1.2 \%$ in the control, $p=0.01)$. Cultures grown in CnT-PR contained $2.7 \% \pm 1.1 \% \mathrm{~K} 12^{+}$cells, and cultures grown in ESCM contained $1.8 \% \pm 0.6 \% \mathrm{~K}^{+} 2^{+}$cells $(p>0.05$; Fig. 5A, D). Overall, such small proportions of differentiated $\mathrm{K}_{12}{ }^{+}$cells are not clinically significant.

We measured the percentage of p63 $\alpha^{\text {bright }}$ cells in the cultures as a potential indication of the LSC transplantation success. ${ }^{1}$ Cultures grown in CnT-PR and mSHEM contained 
proportions of p63 $\alpha^{\text {bright }}$ cells $(17.8 \% \pm 5.7 \%$ and $16.1 \% \pm$ $0.4 \%$, respectively) similar to those in the explant SHEM Cntl $(22.8 \% \pm 2.4 \%, p>0.05$; Fig. 5B, E). ESCM was the culture condition that produced the least amount of p63 $\alpha^{\text {bright }}$ cells $(6.7 \% \pm 3.6 \%, p=0.04$; Fig. 5B, E).

The expression of PanK and Vim proteins was examined to determine the proportion of epithelial cells $\left(\mathrm{PanK}^{+} / \mathrm{Vim}^{-}\right.$ and $\left.\mathrm{PanK}^{+} / \mathrm{Vim}^{+}\right)$and stromal cells $\left(\mathrm{PanK}^{-} / \mathrm{Vim}^{+}\right)$in the cultures. In general, all the cultures had a high percentage of either $\mathrm{PanK}^{+} / \mathrm{Vim}^{-}$or $\mathrm{PanK}^{+} / \mathrm{Vim}^{+}$cells $(>95 \%$ in all the conditions, $p>0.05$; Fig. 5C, F). Only the ESCM cultures contained a proportion of $\mathrm{PanK}^{-} / \mathrm{Vim}^{+}$stromal cells $(1.6 \% \pm 0.8 \%)$ that was significantly larger than that of the control $(0.6 \% \pm 0.3 \%, p=0.04)$.

\section{Discussion}

To have a standardized LSC therapy using cultivated LSCs, there is the need for an optimized and xenobiotic-free culture system. In the present study, we investigated three different base culture media in their ability to support the expansion of LSCs using a standard set of quantifiable criteria. The optimal formulation of each of the three base medium was determined before these three base media were directly compared with one another.

CnT-PR appears to be less efficient in supporting the growth of LSCs in vitro than the standard SHEM. We hypothesized that the LSC growth efficiency could be improved by supplementing a higher level of HS. We found that CnTPR supplemented with 5\% HS (CnT-PR 5) was better than CnT-PR supplemented with $1 \%$ HS in maintaining the LSC phenotype of most LSC cultures. However, the CnT-PR 5 medium was still less efficient than the standard SHEM in supporting the expansion of the LSC population.

LSCs and limbal stromal niche cells have been cultured as spheres in three-dimensional (3D) Matrigel ${ }^{\circledR}$ using ESCM to prevent differentiation and to maintain the clonal growth of LSCs. ${ }^{6}$ This method was excellent in maintaining the close association between LSCs and limbal stromal cells, but LSC proliferation was not optimal. ${ }^{6}$ This finding is consistent with our own: when ESCM alone was used, the LSC proliferation rate was low, and some areas of outgrowth contained spindled, fibroblast-like cells. ESCM may favor the growth of these fibroblast-like stromal cells over the epithelial cells; this possibility is supported by the increase in the number of small, stromal-like $\mathrm{PanK}^{-} / \mathrm{Vim}^{+}$cells. In a three-dimensional (3D) system such as Matrigel, the association between stromal cells and LSCs helped in the maintenance of LSC phenotype, which is similar to the effect of the in vivo niche. ${ }^{6}$ However, in a 2D system, in which both LSCs and stromal cells are plated together on a culture dish (e.g., a 2D system such as an explant culture on AM), there might be competition for the growth space and growth factors that favor the growth of stromal cells over the epithelial cells.

The mSHEM without DMSO and cholera toxin provided the most efficient and consistent growth of LSCs. Cholera toxin is well known for accelerating the cellular growth rate of human keratinocytes in culture by increasing the intracellular concentration of cyclic AMP; this intracellular second signaling messenger regulates cell proliferation. ${ }^{12}$ However, cholera toxin is obtained from bacteria and manufactured using animal components; moreover, it has been reported to have side effects in humans. ${ }^{13}$ Isoproterenol is a nonselective beta-adrenergic agonist that has been previously shown, at a concentration of $1 \mu \mathrm{g} / \mathrm{mL}$, to efficiently replace cholera toxin and thus enhance cell proliferation. $^{8}$ DMSO enhances the permeability of the lipid cell membranes during the cell culture period ${ }^{14}$; however, when DMSO has been included in stem cell cultures used for bone marrow stem cell transplantation, there have been reports of toxic side effects such as cardiovascular and respiratory issues due to DMSOs dose-dependent vasoconstrictor effect. ${ }^{15}$ To minimize the potential toxicity of DMSO, different cell culture strategies were evaluated; among all conditions tested, the removal of DMSO in combination with the addition of isoproterenol resulted in the most efficient LSC growth. This strategy would signify a safer way to culture the LSCs for future transplantation by potentially diminishing the potential toxic effects of DMSO and cholera toxin to the recipient.

EGF appears to have different effects on LSCs in culture. Some studies have shown the importance of EGF in promoting LSC proliferation ${ }^{16}$; other studies have found an increase in LSC motility and a decrease in the induction of K12 expression in the presence of EGF. ${ }^{17}$ EGF has also been shown to increase survival and colony-forming efficiency, but not necessarily the LSC growth rate. ${ }^{18}$ Moreover, longterm cultures in the presence of EGF have not survived more than 3 months. ${ }^{19}$ When EGF concentration in cultures was reduced five times from 2.0 to $0.4 \mathrm{ng} / \mathrm{mL}$, we found a more homogenous LSC-like cell morphology and a slight increase in the percentages of small cells and p63 $\alpha^{\text {bright }}$ cells without a significant decrease in the proliferation rate. Therefore, shortterm use of reduced EGF concentrations may favor cell survival and maintain the same degree of proliferation.

In conclusion, a robust xenobiotic-free culture system that can consistently support a sufficient expansion and maintain the undifferentiated state of LSCs is highly desired to achieve a successful reconstruction of a normal corneal epithelial surface in eyes with severe or total LSCD. Herein, we present a comparative analysis on different xenobioticfree culture media using a set of quantitative criteria to standardize the cultivated LSC characterization. A mSHEMbased xenobiotic-free medium can consistently support LSC expansion from different limbal donor tissues.

\section{Acknowledgments}

This work was supported by the National Eye Institute (R01EY021797 and 5P30EY000331), California Institute for Regenerative Medicine (TR2-01768, BF1-01768 and CLIN1-08686), and an unrestricted grant from Research to Prevent Blindness. We thank Elfren Baclagon for technical support.

\section{Disclosure Statement}

No competing financial interests exist.

\section{References}

1. Rama, P., Matuska, S., Paganoni, G., Spinelli, A., De Luca, M., and Pellegrini, G. Limbal stem-cell therapy and longterm corneal regeneration. N Engl J Med 363, 147, 2010. 
2. Sangwan, V.S., Basu, S., Vemuganti, G.K., Sejpal, K., Subramaniam, S.V., Bandyopadhyay, S., Krishnaiah, S., Gaddipati, S., Tiwari, S., and Balasubramanian, D. Clinical outcomes of xeno-free autologous cultivated limbal epithelial transplantation: a 10-year study. $\mathrm{Br} \mathrm{J}$ Ophthalmol 95, 1525, 2011.

3. Shortt, A.J., Bunce, C., Levis, H.J., Blows, P., Dore, C.J., Vernon, A., Secker, G.A., Tuft, S.J., and Daniels, J.T. Three-year outcomes of cultured limbal epithelial allografts in aniridia and Stevens-Johnson syndrome evaluated using the Clinical Outcome Assessment in Surgical Trials assessment tool. Stem Cells Transl Med 3, 265, 2014.

4. Zakaria, N., Koppen, C., Van Tendeloo, V., Berneman, Z., Hopkinson, A., and Tassignon, M.J. Standardized limbal epithelial stem cell graft generation and transplantation. Tissue Eng Part C Methods 16, 921, 2010.

5. Zakaria, N., Possemiers, T., Dhubhghaill, S.N., Leysen, I., Rozema, J., Koppen, C., Timmermans, J.P., Berneman, Z., and Tassignon, M.J. Results of a phase I/II clinical trial: standardized, non-xenogenic, cultivated limbal stem cell transplantation. J Transl Med 12, 58, 2014.

6. Xie, H.T., Chen, S.Y., Li, G.G., and Tseng, S.C. Isolation and expansion of human limbal stromal niche cells. Invest Ophthalmol Vis Sci 53, 279, 2012.

7. Zhang, S., Liu, Z., Su, G., and Wu, H. Comparative Analysis of KnockOut Serum with Fetal Bovine Serum for the In Vitro Long-Term Culture of Human Limbal Epithelial Cells. J Ophthalmol 2016, 7304812, 2016.

8. Ghoubay-Benallaoua, D., Pecha, F., Goldschmidt, P., Fialaire-Legendre, A., Chaumeil, C., Laroche, L., and Borderie, V.M. Effects of isoproterenol and cholera toxin on human limbal epithelial cell cultures. Curr Eye Res 37, 644, 2012.

9. Zhang, T., Yam, G.H., Riau, A.K., Poh, R., Allen, J.C., Peh, G.S., Beuerman, R.W., Tan, D.T., and Mehta, J.S. The effect of amniotic membrane de-epithelialization method on its biological properties and ability to promote limbal epithelial cell culture. Invest Ophthalmol Vis Sci 54, 3072, 2013.

10. Gonzalez, S., and Deng, S.X. Presence of native limbal stromal cells increases the expansion efficiency of limbal stem/progenitor cells in culture. Exp Eye Res 116, 169, 2013.

11. Di Iorio, E., Barbaro, V., Ferrari, S., Ortolani, C., De Luca, M., and Pellegrini, G. Q-FIHC: quantification of fluorescence immunohistochemistry to analyse p63 isoforms and cell cycle phases in human limbal stem cells. Microsc Res Tech 69, 983, 2006.
12. Okada, N., Kitano, Y., and Ichihara, K. Effects of cholera toxin on proliferation of cultured human keratinocytes in relation to intracellular cyclic AMP levels. J Invest Dermatol 79, 42, 1982.

13. Spangler, B.D. Structure and function of cholera toxin and the related Escherichia coli heat-labile enterotoxin. Microbiol Rev 56, 622, 1992.

14. Notman, R., Noro, M., O’Malley, B., and Anwar, J. Molecular basis for dimethylsulfoxide (DMSO) action on lipid membranes. J Am Chem Soc 128, 13982, 2006.

15. Windrum, P., Morris, T.C., Drake, M.B., Niederwieser, D., Ruutu, T.; EBMT Chronic Leukaemia Working Party Complications Subcommittee. Variation in dimethyl sulfoxide use in stem cell transplantation: a survey of EBMT centres. Bone Marrow Transplant 36, 601, 2005.

16. Trosan, P., Svobodova, E., Chudickova, M., Krulova, M., Zajicova, A., and Holan, V. The key role of insulin-like growth factor I in limbal stem cell differentiation and the corneal wound-healing process. Stem Cells Dev 21, 3341, 2012.

17. Wilson, S.E., He, Y.G., Weng, J., Zieske, J.D., Jester, J.V., and Schultz, G.S. Effect of epidermal growth factor, hepatocyte growth factor, and keratinocyte growth factor, on proliferation, motility and differentiation of human corneal epithelial cells. Exp Eye Res 59, 665, 1994.

18. Rheinwald, J.G., and Green, H. Epidermal growth factor and the multiplication of cultured human epidermal keratinocytes. Nature 265, 421, 1977.

19. Miyashita, H., Yokoo, S., Yoshida, S., Kawakita, T., Yamagami, S., Tsubota, K., and Shimmura, S. Long-term maintenance of limbal epithelial progenitor cells using rho kinase inhibitor and keratinocyte growth factor. Stem Cells Transl Med 2, 758, 2013.

Address correspondence to: Sophie X. Deng, MD, PhD Stein Eye Institute University of California, Los Angeles 200 Stein Plaza Los Angeles, CA 90095

E-mail: deng@jsei.ucla.edu

Received: September 26, 2016

Accepted: February 8, 2017

Online Publication Date: March 24, 2017 